\title{
From Distant Stars to Dental Chairs: An Update on Plasma Needle
}

\author{
Vandana B L* \\ Department of Pedodontics \& Preventive Dentistry, KLE Society’s Institute of Dental sciences, Opp to CMTI, Yahswanthpur Suburb, \\ Tumkur Road, Bangalore \\ *Corresponding author: Vandana_bl@yahoo.co.in
}

Received October 11, 2014; Revised October 17, 2014; Accepted December 18, 2014

\begin{abstract}
There is an uncontrolled and unwanted damage to the structure of tooth, whenever any mechanical or a chemical procedure is applied in order to treat a dental cavity or a carious tooth. This led to the development of plasma needle in the field of dentistry. This unique and unprecedented technology, which, though commonly used in medicine for disinfection and sterilization, as well as undertaking decontamination in biological warfare has a tremendous potential in the dental field too. The present article discusses the technology, with special emphasis on its operative details and the useful applications of the same.
\end{abstract}

Keywords: plasma, non thermal atmospheric plasma, plasma needle

Cite This Article: Vandana B L, "From Distant Stars to Dental Chairs: An Update on Plasma Needle." International Journal of Dental Sciences and Research, vol. 2, no. 6B (2014): 19-20. doi: 10.12691/ijdsr-2-6B-6.

\section{Introduction}

Plasma medicine is an innovative and emerging field combining plasma physics, life sciences and clinical medicine to use physical plasma for therapeutic applications. It has been used for sterilization of medical equipment, packaging in the food industry, implants, blood coagulation, wound healing etc [1]. This is partly due to their high bactericidal effectiveness and partly due to their easy access into narrow and confined spaces. Plasma is a collection of stripped particles. When electrons are stripped from atoms and molecules, those partials change state and become plasma [2,3]. In recent years, cold (less than $40{ }^{\circ} \mathrm{C}$ at the point of application) atmospheric plasma sources have been developed that provide the possibility to extend plasma treatment to living tissue [4]. The present article emphasise the use of plasma needle in dentistry.

\subsection{History}

The British physicist Sir William Crookes identified the fourth state of matter in 1879, but it was not called "plasma” until Irving Langmuir, an American chemist, applied the name in 1929. Plasmas are naturally energetic because stripping electrons takes constant energy. If the energy dissipates, the electrons reattach and the plasma particles become a gas once again. Unlike ordinary matter, plasmas can exist in a wide range of temperatures without changing state. In dentistry it is used as a plasma needle to generate a non-thermal atmospheric microplasma. The plasma needle was tested first in 2002 by Dr. I.R. Eva Stoffels-Adamowicz at the Eindhoven University of Technology $[5,6]$.

\subsection{Plasma Needle}

The plasma needle consists of a tungsten wire $(0.3 \mathrm{~mm}$ diameter) with a sharp tip at the end, confined in a Perspex tube (Figure 1) (4 mm inner diameter) [7,8]. The Perspex tube is filled with helium. Helium flows through the plastic tube at a rate of 2 lt min. A radio-frequency (RF) voltage is applied to the needle at $13.56 \mathrm{MHz}$. The signal is produced by a waveform generator and amplified by a RF amplifier. The dissipated power is monitored using a probe connected via a dual-directional coupler to a power meter. The apparatus applies small amounts of electricity to a non-toxic gas through a "narrow slit chamber" to generate plasma with a brush-like shape which is cool to touch. It glides across the surface of the tooth, killing bacteria and preparing the dentin and enamel. Gas heating is directly related to plasma power, so using higher powers in combination with short distances should be avoided. Typical average power is $0.5-2 \mathrm{~W}$. Right dose can be administered by adjusting plasma power, distance to the substrate, treatment time and gas composition [9].

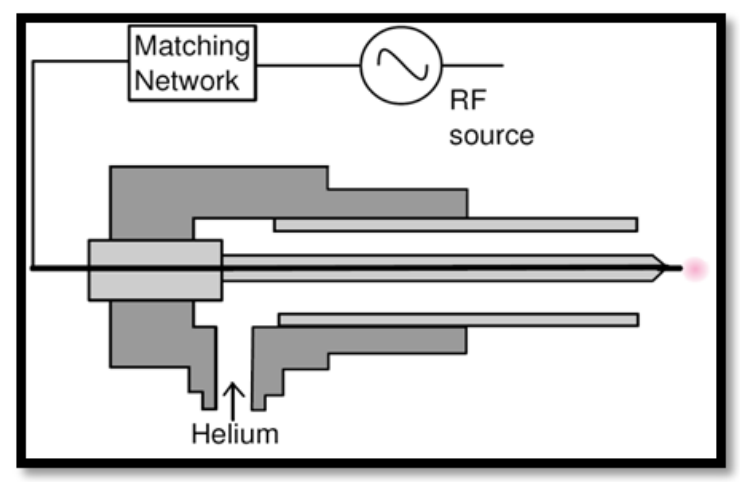

Figure 1. 


\subsection{Applications in Dentistry}

Dental applications have emerged because a new version of plasma technology, called "non-thermal atmospheric plasmas,” permits surface preparation in open air at room temperature [10].

Cavity preparation: The plasma needle produces short-lived chemical species in the gas phase. These can interact on a tooth's surface, and they can dissolve into a liquid. Unlike liquid rinses with bactericidal ingredients that linger in the mouth after treatment, the plasma needle produces bactericidal agents locally. These particles can reach the inside of the cavity and fissure spaces [10]. Once the treatment is completed, no excess radicals remain, due to recombination among themselves or by reacting with ambient air and water molecules. Thus, the bactericidal agents have a naturally short lifetime, which is an attractive feature [8]. Effective in children as there is no drilling, painless fear free dentistry.

Composite restorations: Studies has shown that plasma treatment increases bonding strength at the dentin/ composite interface by roughly 60 percent. This interfacebonding enhances composite performance, durability, and longevity. Dr. Kong and his colleagues investigated the plasma treatment effects on dental composite restoration for improved interface properties and their experimental results showed that plasma brush treatment can modify the dentin surface and thus increase the dentin/adhesive interfacial bonding. The solution is to introduce bonds that depend on surface chemistry rather than surface porosity [12]. Yavrich et al studied the effects of plasma treatment on the shear bond strength between fiber reinforced composite posts and resin composite for core buildup and concluded that plasma treatment appeared to increase the tensile-shear bond strength between post and composite [13].

Bleaching: $\mathrm{OH}$ is crucial in tooth bleaching. $\mathrm{OH}$ possesses an unpaired electron, so it is unstable and very reactive. It captures an electron from pigments disrupting their structures and bleaching occurs. Generally, $\mathrm{OH}$ is generated from $\mathrm{H}_{2} \mathrm{O}_{2}$ by interaction with a catalyst that contains divalent cations. The amount of $\mathrm{OH}$ nearly doubled after plasma treatment for 1 minute, compared to conventional methods [14]. Lee et al in his study concluded that tooth bleaching is enhanced by plasmas, mainly due to the in situ production of hydroxyl free radicals [15].

Disinfection of root canals: Lu et al used a plasma-jet device, which could generate plasma inside the root canal. The plasma could be directed manually by a user to place it into root canal for disinfection without causing any painful sensation. When Helium/Oxygen(20\%) is used as working gas, the rotational and vibrational temperatures of the plasma are about $300 \mathrm{~K}$ and $2700 \mathrm{~K}$, respectively. The peak discharge current is about $10 \mathrm{~mA}$. Experiment results showed that it can efficiently kill enterococcus faecalis, one of the main bacterium causing failure of root-canal [11].

Other uses: It can be used for sterilization of instruments, treatment of periodontal pockets, biofilms. Plasma using is known to create active species such as hydroxyl radicals, ozone, hydrogen peroxide and super oxide radicals. These reactive species have shown to disrupt the cell membrane of microorganisms and cause cell death. Sladek in his study tested plasma for its antimicrobial activity against biofilm cultures of Streptococcus mutans and showed that plasma has a significant antibacterial activity [16].

\section{Conclusion}

Painless dentistry would be a huge advance in the general consensus. Dental lasers have attempted to address this concern but have proven expensive and slow. The problem of fear is more an issue with children and underserved communities. The plasma brush may serve as an extremely valuable tool among pedodontists and dentists in less-serviced communities as it is painless, noiseless and effective. Although plasma technology isn't an end-all to all the techniques we perform, it could well become a valuable tool.

\section{References}

[1] Deilmann M, Halfmann H, Bibinov N, Wunderlich J and Awakowicz P. Low-pressure microwave plasma sterilization of polyethylene terephthalate bottles. J. Food Prot 2008;71: 21192123.

[2] Fridman G, Gutsol A, Shekhter A B, Vasilets V N and Fridman A. Applied plasma medicine. Plasma Process. Polym 2008; 5: 503533.

[3] Moreau M, Orange N and Feuilloley M G J. Non-thermal plasma technologies: new tools for bio-decontamination. Biotechnol. Adv. 2008; 26: 610-617.

[4] Deng X, Shi J J and Kong M G. Protein destruction by a helium atmospheric pressure glow discharge: capability and mechanisms. J. Appl. Phys. 2007; 101: 074701.

[5] Farin G and Grund K E. Technology of argon plasma coagulation with particular regard to endoscopic applications. Endosc. Surg. Allied Technol. 1994; 2: 71-77.

[6] Stoffels E, Flikweert AJ, Stoffels WW, Kroesen GMW. Plasma needle: a non destructive atmospheric plasma source for fine surface treatment of biomaterials. Plasma Sources Sci. Technol. 11 (2002) 383-388.

[7] Sladek REJ and Stoffels E. Deactivation of Escherichia coli by the plasma needle. J Phys. D: Appl. Phys 2005; 38: 1716-1721.

[8] Walrayen R, Sladek REJ, Stoffels E, Tielbeek PJA, Koolhoven RA. Plasma Treatment of Dental Cavities: A Feasibility Study. IEEE Transactions on Plasma Science 2004; 32(4): 1540-1543.

[9] Stoffels E, Kieft IE, Sladek REJ et.al. Plasma needle for in vivo medical treatment: recent developments and perspectives. Plasma Sources Sci.Technol 2006; 15:169-180.

[10] Sladek REJ, Stoffels E. Plasma treatment of dental cavities: A feasibility study. IEEE transactions on plasma science vol 32, no 4; 2004.

[11] Plasma needle: The future of Dentistry. Somya Govil, Vishesh Gupta,IJBAMR Issue-2, Vol.-1, P. 143-147, 2012.

[12] M G Kong, G Kroesen, G Morfill, T Nosenko, T Shimizu, J van Dijk and J L Zimmermann. Plasma medicine: an introductory review. New J. Phys. 2009; 11: 115012.

[13] Yavirach P, Chaijareenont P, Boonyawan D, Pattamapun K, Tunma S, Takahashi H, Arksornnukit M. Effects of plasma treatment on the shear bond strength between fiber reinforced composite posts and resin composite for core build-up. Dental Materials Journal 2009; 28(6): 686-692.

[14] HyunWoo Lee, Gon Jun.Tooth Bleaching with Nonthermal Atmospheric Pressure Plasma. JOE - Volume 35, Number 4, April 2009.

[15] Van den Bedem LJM, Sladek REJ, Steinbuch M, Adamowicz ES. Plasma treatment of S. mutans biofilms cultured in a simulated dental cavity model. XXVIIth ICPIG, Eindhoven, the Netherlands, 18-22 July, 2005.

[16] Sladek, R., Filoche, S., Sissons, C. and Stoffels, E. (2007). Treatment of Streptococcus mutans biofilms with a nonthermal atmospheric plasma. Letters in Applied Microbiology, 45: 318323. 